\title{
Ulam-Hyers Stability for MKC Mappings via Fixed Point Theory
}

\author{
Anisa Mukhtar Hassan, ${ }^{1}$ Erdal Karapınar, ${ }^{2,3}$ and Hamed H. Alsulami ${ }^{3}$ \\ ${ }^{1}$ King Abdulaziz University, Jeddah, Saudi Arabia \\ ${ }^{2}$ Department of Mathematics, Attlim University, Incek, 06836 Ankara, Turkey \\ ${ }^{3}$ Nonlinear Analysis and Applied Mathematics Research Group (NAAM), King Abdulaziz University, Jeddah, Saudi Arabia
}

Correspondence should be addressed to Erdal Karapınar; erdalkarapinar@yahoo.com

Received 7 January 2016; Accepted 5 April 2016

Academic Editor: Liviu Cădariu

Copyright (C) 2016 Anisa Mukhtar Hassan et al. This is an open access article distributed under the Creative Commons Attribution License, which permits unrestricted use, distribution, and reproduction in any medium, provided the original work is properly cited.

We consider some extension of MKC mappings in the framework of complete dislocated metric spaces. Besides the theoretical results, we also consider some illustrative examples. Further, we state and prove that our main results improved the related results in the frame of generalized Ulam-Hyers stability theory.

\section{Introduction and Preliminaries}

At the beginning of the nineteenth century, Fréchet [1] explicated the concept of "distance" formally by introducing the notion of metric. This conception has been generalized, extended, and improved in several ways, such as fuzzy metric, quasi-metric, partial metric, G-metric, D-metric, b-metric, 2-metric, ultrametric, and modular metric. Among them, the concept of dislocated metric, defined by Hitzler [2], deserves a special interest due to its application potential in several quantitative sciences, in particular, computer science. The concept of dislocated metric spaces was rediscovered by Amini-Harandi [3] as a "metric-like space."

In this paper, we continue to use dislocated metric space instead of metric-like space.

Definition 1 (see [2]). For a nonempty set $M$, a dislocated metric is a function $\rho: M \times M \rightarrow \mathbb{R}_{0}^{+}$such that, for all $\xi, \eta, \zeta \in M$,

$$
\begin{aligned}
& \left(\rho_{1}\right) \text { if } \rho(\xi, \eta)=0 \text {, then } \xi=\eta ; \\
& \left(\rho_{2}\right) \rho(\xi, \eta)=\rho(\eta, \xi) ; \\
& \left(\rho_{3}\right) \rho(\xi, \eta) \leq \rho(\xi, \zeta)+\rho(\zeta, \eta) .
\end{aligned}
$$

Moreover, the pair $(M, \rho)$ is called dislocated metric space (DMS).
The topology of dislocated spaces and basic topological properties (convergence, completeness, etc.) was also considered in [2]. Among them, let us recall the essential notions. Each dislocated metric $\rho$ on a nonempty set $M$ has a topology $\tau_{\rho}$ that was generated by the family of open balls

$$
\begin{aligned}
O_{\varepsilon}(\xi)=\{\eta \in M:|\rho(\xi, \eta)-\rho(\xi, \xi)|<\varepsilon\} & \\
& \forall \xi \in M, \varepsilon>0 .
\end{aligned}
$$

In context of the DMS $(M, \rho)$, a sequence $\left\{\xi_{n}\right\}$ converges to a point $\xi \in M$ if the following limit exists (and is finite):

$$
\lim _{n \rightarrow \infty} \rho\left(\xi_{n}, \xi\right)=\rho(\xi, \xi) .
$$

Moreover, a sequence $\left\{\xi_{n}\right\}$ is said to be Cauchy if the limit

$$
L_{1}=\lim _{n \rightarrow \infty} \rho\left(\xi_{n}, \xi_{m}\right)
$$

exists and is finite. Furthermore, if $L_{1}=0$ in (3), then we say that $\left\{\xi_{n}\right\}$ is a 0 -Cauchy sequence.

As it is expected, a pair $(M, \rho)$ is called complete DMS if, for each Cauchy sequence $\left\{\xi_{n}\right\}$, there is some $\xi \in M$ such that

$$
L_{2}=\lim _{n \rightarrow \infty} \rho\left(\xi_{n}, \xi\right)=\rho(\xi, \xi)=\lim _{n \rightarrow \infty} \rho\left(\xi_{n}, \xi_{m}\right) .
$$

Analogously, a pair $(M, \rho)$ is called 0 -complete DMS if, for each 0 -Cauchy sequence $\left\{\xi_{n}\right\}$, it converges to a point $\xi \in$ $M$ such that $L_{2}=0$ in (4). 
Let $(M, \rho)$ and $(K, \sigma)$ be DMSs. A mapping $T: M \rightarrow K$ is called continuous if

$$
\lim _{n \rightarrow \infty} \rho\left(\xi_{n}, \xi\right)=\rho(\xi, \xi)=\lim _{n, m \rightarrow \infty} \rho\left(\xi_{n}, \xi_{m}\right)
$$

then, we have

$$
\lim _{n \rightarrow \infty} \rho\left(T \xi_{n}, T \xi\right)=\rho(T \xi, T \xi)=\lim _{n, m \rightarrow \infty} \rho\left(T \xi_{n}, T \xi_{m}\right) .
$$

Remark 2. Each partial metric space is a DMS. Notice also that every metric is necessarily partial metric.

Definition 3 (see [3]). Let $(M, \rho)$ be a DMS and let $S$ be a subset of $M$. One says that $S$ is an open subset of $M$, if for all $\xi \in M$ there exists $r>0$ such that $O_{r}(\xi) \subseteq S$. Also, $F \subseteq X$ is a closed subset of $M$ if $(M \backslash F)$ is an open subset of $M$.

Lemma 4 (see [4]). For a DMS $(M, \rho)$, one has the following observations:

(A) If $\rho(\xi, \eta)=0$, then $\rho(\xi, \xi)=\rho(\eta, \eta)=0$.

(B) For a sequence $\left\{\xi_{n}\right\}$ with $\lim _{n \rightarrow \infty} \rho\left(\xi_{n}, \xi_{n+1}\right)=0$, one has

$$
\lim _{n \rightarrow \infty} \rho\left(\xi_{n}, \xi_{n}\right)=\lim _{n \rightarrow \infty} \rho\left(\xi_{n+1}, \xi_{n+1}\right)=0
$$

(C) If $\xi \neq \eta$, then $\rho(\xi, \eta)>0$.

(D) $\rho(\xi, \xi) \leq(2 / n) \sum_{i=1}^{i=n} \rho\left(\xi, \xi_{i}\right)$ holds for all $\xi_{i}, \xi \in M$, where $1 \leq i \leq n$.

(E) Let $V$ be a closed subset of $M$ and let $\left\{\xi_{n}\right\}$ be a sequence in $V$. If $\xi_{n} \rightarrow \xi$ as $n \rightarrow \infty$, then $\xi \in V$.

(F) For a sequence $\left\{\xi_{n}\right\}$ in $M$ such that $\xi_{n} \rightarrow \xi$ as $n \rightarrow \infty$ with $\rho(\xi, \xi)=0$, then $\lim _{n \rightarrow \infty} \rho(\xi, \eta)=\rho(\xi, \eta)$ for all $\eta \in M$.

Definition 5 (see [5]). Let $M$ be a nonempty set and let $\alpha$ : $M \times M \rightarrow[0, \infty)$ be a mapping. A self-mapping $T: M \rightarrow M$ is called $\alpha$-admissible if the following implications hold:

$$
\begin{aligned}
\alpha(\xi, \eta) & \geq 1 \Longrightarrow \\
\alpha(T \xi, T \eta) & \geq 1
\end{aligned}
$$

$$
\forall \xi, \eta \in M
$$

An $\alpha$-admissible $T$ is said to be triangular $\alpha$-admissible if

$$
\begin{aligned}
& \alpha(\xi, \eta) \geq 1, \alpha(\eta, \zeta) \geq 1 \Longrightarrow \\
& \alpha(\xi, \zeta) \geq 1,
\end{aligned}
$$

$$
\forall \xi, \eta, \zeta \in M
$$

The letter $\mathbb{N}$ represents the set of positive integers and $\mathbb{N}_{0}=\mathbb{N} \cup\{0\}$. Further, the nonnegative real numbers will be denoted by $\mathbb{R}_{0}^{+}=[0, \infty)$.
1.1. (c)-Comparison Functions. Let $\Psi$ be the family of nondecreasing functions $\psi: \mathbb{R}_{0}^{+} \rightarrow \mathbb{R}_{0}^{+}$satisfying the following conditions:

$\left(\Psi_{1}\right) \sum_{n=1}^{+\infty} \psi^{n}(t)<\infty$ for all $t>0$, where $\psi^{n}$ is the $n$th iterate of $\psi$.

$$
\left(\Psi_{2}\right) \psi^{-1}(0)=0 \text {. }
$$

It is well known that if $\psi$ is a (c)-comparison function, then $\psi(t)<t$ for any $t>0$ and $\psi$ is continuous at 0 (see, e.g., [6]).

\section{Fixed Point Theorems for $(\alpha, \psi)$-Meir-Keeler Contractions on Dislocated Metric Spaces}

2.1. $(\alpha, \psi)$-Meir-Keeler Contractive Mapping. We introduce the following notion which is an improved version of MeirKeeler contractive mapping.

Definition 6. Let $(M, \rho)$ be a DMS. A self-mapping $T: M \rightarrow$ $M$ is called $(\alpha, \psi)$-Meir-Keeler contractive (in brief, $(\alpha, \psi)$ $\mathrm{MKC}$ ) if there exist two functions $\psi \in \Psi$ and $\alpha: M \times M \rightarrow \mathbb{R}_{0}^{+}$ satisfying the following condition.

For each $\varepsilon>0$, there exists $\delta>0$ such that

$$
\begin{aligned}
& \varepsilon \leq \psi(\rho(\xi, \eta))<\varepsilon+\delta \\
& \text { implies } \alpha(\xi, \eta) \rho(T \xi, T \eta)<\varepsilon .
\end{aligned}
$$
have

Notice that, for $(\alpha, \psi)$-MKC mapping $T: M \rightarrow M$, we

$$
\alpha(\xi, \eta) \rho(T \xi, T \eta) \leq \psi(\rho(\xi, \eta)) \text {, for any } \xi, \eta \in M \text {. }
$$

In what follows, we will state and prove the first main result of this section.

Theorem 7. Suppose that $(M, \rho)$ is a complete DMS and a selfmapping $T: M \rightarrow M$ is $(\alpha, \psi)-M K C$. Assume also that

(i) $T$ is $\alpha$-admissible;

(ii) there exists $\xi_{0} \in M$ such that $\alpha\left(\xi_{0}, T \xi_{0}\right) \geq 1$;

(iii) $T$ is continuous.

Then, there exists $\theta \in M$ such that $T \theta=\theta$.

Proof. Due to (ii), there exists $\xi_{0} \in M$ such that $\alpha\left(\xi_{0}, T \xi_{0}\right) \geq$ 1 . We define an iterative sequence $\left\{\xi_{n}\right\}$ in $M$ as

$$
\xi_{n+1}=T \xi_{n}, \quad \forall n \in \mathbb{N}_{0} .
$$

Notice that if there exists some $k \in \mathbb{N}$ such that $\xi_{k}=\xi_{k+1}$, then the proof is completed since $\theta=\xi_{k}=\xi_{k+1}=T \xi_{k}=T \theta$.

So, we assume that $\xi_{n} \neq \xi_{n+1}$, for all $n$. Due to Lemma 4 part (C), for $\xi_{n} \neq \xi_{n+1}$, we have

$$
\rho\left(\xi_{n}, \xi_{n+1}\right)>0, \quad \forall n \in \mathbb{N}_{0} .
$$

Since $T$ is $\alpha$-admissible, again by (ii), we get

$$
\begin{gathered}
\alpha\left(\xi_{0}, \xi_{1}\right)=\alpha\left(\xi_{0}, T \xi_{0}\right) \geq 1 \Longrightarrow \\
\alpha\left(T \xi_{0}, T \xi_{1}\right)=\alpha\left(\xi_{1}, \xi_{2}\right) \geq 1 .
\end{gathered}
$$


By repeating this process, we derive that

$$
\alpha\left(\xi_{n}, \xi_{n+1}\right) \geq 1, \quad \forall n \in \mathbb{N}_{0} .
$$

Regarding (12) and (15) together with the assumption of the theorem that $T$ is an $(\alpha, \psi)$-MKC mapping, for each $n \in \mathbb{N}$, we find

$$
\begin{aligned}
\rho\left(\xi_{n}, \xi_{n+1}\right) & =\rho\left(T \xi_{n-1}, T \xi_{n}\right) \\
& \leq \alpha\left(\xi_{n-1}, \xi_{n}\right) \rho\left(T \xi_{n-1}, T \xi_{n}\right) \\
& \leq \psi\left(\rho\left(\xi_{n-1}, \xi_{n}\right)\right) .
\end{aligned}
$$

Taking (13) and the property of $\psi$ into account, the above inequalities yield that

$$
\rho\left(\xi_{n}, \xi_{n+1}\right) \leq \psi\left(\rho\left(\xi_{n-1}, \xi_{n}\right)\right)<\rho\left(\xi_{n-1}, \xi_{n}\right)
$$

Eventually, we conclude that $\left\{\rho\left(\xi_{n-1}, \xi_{n}\right)\right\}$ is a nonincreasing and bounded sequence. Hence, there exists $t \in[0, \infty)$ such that

$$
\lim _{n \rightarrow \infty} \rho\left(\xi_{n-1}, \xi_{n}\right)=t
$$

In what follows, we will prove that $t=0$. Suppose, on the contrary, that $t>0$. Since $T$ is an $(\alpha, \psi)$-MKC mapping, for $\varepsilon=\psi(t)>0$, there exist $\delta>0$ and a natural number $m$ such that

$$
\begin{aligned}
& \varepsilon \leq \psi\left(\rho\left(\xi_{m-1}, \xi_{m}\right)\right)<\varepsilon+\delta \\
& \text { implies } \alpha\left(\xi_{m-1}, \xi_{m}\right) \rho\left(T \xi_{m-1}, T \xi_{m}\right)<\varepsilon .
\end{aligned}
$$

By taking (15) into account, we get that

$$
\begin{aligned}
\rho\left(\xi_{m}, \xi_{m+1}\right) & =\rho\left(T \xi_{m-1}, T \xi_{m}\right) \\
& \leq \alpha\left(\xi_{m-1}, \xi_{m}\right) \rho\left(T \xi_{m-1}, T \xi_{m}\right)<\varepsilon \\
& =\psi(t)<t,
\end{aligned}
$$

which is a contradiction since $t=\inf \left\{\rho\left(\xi_{n}, \xi_{n+1}\right): n \in \mathbb{N}\right\}$.

Consequently, we have

$$
\lim _{n \rightarrow \infty} \rho\left(\xi_{n-1}, \xi_{n}\right)=0
$$
is,

Now, we will prove that $\left\{\xi_{n}\right\}$ is a Cauchy sequence. That

$$
\lim _{n \rightarrow \infty} \rho\left(\xi_{n}, \xi_{n+k}\right)=0, \quad \forall k \in \mathbb{N}
$$

First, we observe from (17) that

$$
\rho\left(\xi_{n}, \xi_{n+1}\right) \leq \psi\left(\rho\left(\xi_{n-1}, \xi_{n}\right)\right) \quad \forall n \in \mathbb{N} .
$$

Since $\psi$ is nondecreasing, by iteration, we conclude that

$$
\rho\left(\xi_{n}, \xi_{n+1}\right) \leq \psi^{n}\left(\rho\left(\xi_{0}, \xi_{1}\right)\right) \quad \forall n \in \mathbb{N} \text {. }
$$

Now, by using $\left(\rho_{3}\right),(12),(15)$, and $(24)$, we have the following:

$$
\begin{aligned}
\rho\left(\xi_{n}, \xi_{n+k}\right) & \leq \rho\left(\xi_{n}, \xi_{n+1}\right)+\cdots+\rho\left(\xi_{n+k-1}, \xi_{n+k}\right) \\
& =\sum_{p=n}^{n+k-1} \rho\left(\xi_{p}, \xi_{p+1}\right)=\sum_{p=n}^{n+k-1} \rho\left(T \xi_{p-1}, T \xi_{p}\right) \\
& \leq \sum_{p=n}^{n+k-1} \alpha\left(\xi_{p-1}, \xi_{p}\right) \rho\left(T \xi_{p-1}, T \xi_{p}\right) \\
& \leq \sum_{p=n}^{n+k-1} \psi\left(\rho\left(\xi_{p-1}, \xi_{p}\right)\right) \\
& =\sum_{p=n}^{n+k-1} \psi^{p-1}\left(\rho\left(\xi_{0}, \xi_{1}\right)\right) \\
& \leq \sum_{p=n}^{\infty} \psi^{p-1}\left(\rho\left(\xi_{0}, \xi_{1}\right)\right) .
\end{aligned}
$$

Letting $n \rightarrow \infty$ in the above inequality, we derive expression (22). That is, the iterative sequence $\left\{\xi_{n}\right\}$ is Cauchy in the DMS $(M, \rho)$.

Since $(M, \rho)$ is a complete DMS, then there exists $\theta \in M$ such that

$$
\lim _{n \rightarrow \infty} \rho\left(\xi_{n}, \theta\right)=\rho(\theta, \theta)=\lim _{n \rightarrow \infty} \rho\left(\xi_{n}, \xi_{n+k}\right)=0 .
$$

As $T$ is continuous, then we deduce that

$$
\begin{aligned}
\lim _{n \rightarrow \infty} \rho\left(\xi_{n+1}, T \theta\right) & =\lim _{n \rightarrow \infty} \rho\left(T \xi_{n}, T \theta\right)=\rho(T \theta, T \theta) \\
& =\lim _{n \rightarrow \infty} \rho\left(T \xi_{n}, T \xi_{n+k}\right)=0 .
\end{aligned}
$$

Since $\xi_{n+1} \rightarrow u$ as $n \rightarrow \infty$ and $\rho(\theta, \theta)=0$, then, by using Lemma 4, we get

$$
\lim _{n \rightarrow \infty} \rho\left(\xi_{n+1}, T \theta\right)=\rho(\theta, T \theta) .
$$

From (27) and (28), we derive that $\rho(\theta, T \theta)=0$. By $\left(\rho_{1}\right)$, we conclude that $T \theta=\theta$.

Theorem 8. Suppose that $(M, \rho)$ is a complete DMS and a selfmapping $T: M \rightarrow M$ is $(\alpha, \psi)-M K C$. Assume also that

(i) $T$ is $\alpha$-admissible;

(ii) there exists $\xi_{0} \in M$ such that $\alpha\left(\xi_{0}, T \xi_{0}\right) \geq 1$;

(iii) if $\left\{\xi_{n}\right\}$ is a sequence in $M$ such that $\alpha\left(\xi_{n}, \xi_{n+1}\right) \geq 1$ for all $n$ and $\xi_{n} \rightarrow \xi \in M$ as $n \rightarrow \infty$, then $\alpha\left(\xi_{n}, \xi\right) \geq 1$ for all $n$.

Then, there exists $\theta \in M$ such that $T \theta=\theta$.

Proof. By following the lines in the proof of Theorem 7, we deduce that there exists a Cauchy sequence $\left\{\xi_{n}\right\}$. Since $M$ is complete, it converges to some $\theta \in M$. On the other hand, from (15) and (iii), we have

$$
\alpha\left(\xi_{n}, \theta\right) \geq 1, \quad \forall n .
$$


By using $\left(\rho_{3}\right)$ and (29) with the assumption of the theorem that $T$ is an $(\alpha-\psi)$-MKC mapping, we obtain

$$
\begin{aligned}
\rho(T \theta, \theta) & \leq \rho\left(T \theta, T \xi_{n}\right)+\rho\left(\xi_{n+1}, \theta\right) \\
& \leq \alpha\left(\xi_{n}, \theta\right) \rho\left(T \xi_{n}, T \theta\right)+\rho\left(\xi_{n+1}, \theta\right) \\
& \leq \psi\left(\rho\left(\xi_{n}, \theta\right)\right)+\rho\left(\xi_{n+1}, \theta\right) .
\end{aligned}
$$

Since $\psi$ is continuous at $t=0$, by letting $n \rightarrow \infty$ in the inequality above, we find

$$
\rho(T \theta, \theta)=0 .
$$

It yields $T \theta=\theta$ due to $\left(\rho_{1}\right)$.

Example 9. Let $M=[0, \infty)$ endowed with the dislocated metric $\rho(\xi, \eta)=\max \{x, y\}$ for all $\xi, \eta \in[0, \infty)$. Define $T: M \rightarrow M$ and $\alpha: M \times M \rightarrow \mathbb{R}_{0}^{+}$by

$$
\begin{gathered}
T \xi=\frac{\xi}{2}, \\
\alpha(\xi, \eta)= \begin{cases}1, & \text { if } \xi=\eta=0 ; \\
0, & \text { otherwise. }\end{cases}
\end{gathered}
$$

We can prove easily that $T$ is an $(\alpha-\psi)$-MKC mapping and it is $\alpha$-admissible.

Moreover, there exists $\xi_{0} \in M$ such that $\alpha\left(\xi_{0}, T \xi_{0}\right) \geq 1$. In fact, for $\xi_{0}=0$, we have

$$
\alpha(0, T 0)=1 .
$$

Now, we show that $T$ is continuous. Let

$$
\lim _{n \rightarrow \infty} \rho\left(\xi_{n}, \xi\right)=\rho(\xi, \xi)=\lim _{n, m \rightarrow \infty} \rho\left(\xi_{n}, \xi_{m}\right) .
$$

That is,

$$
\lim _{n \rightarrow \infty} \xi_{n}=\xi
$$

Now, we have that $T$ is continuous since

$$
\begin{aligned}
\lim _{n \rightarrow \infty} \rho\left(T \xi_{n}, T \xi\right) & =\lim _{n \rightarrow \infty} \max \left\{T \xi_{n}, T \xi\right\}=T \xi \\
& =\rho(T \xi, T \xi)=\lim _{n, m \rightarrow \infty} \rho\left(T \xi_{n}, T \xi_{m}\right) .
\end{aligned}
$$

So all hypotheses of Theorem 7 are satisfied. Consequently, $T$ has a fixed point. Notice that $\xi=0$ is a fixed point of $T$.

In the following example, a self-mapping $T$ is not continuous.

Example 10. Let $M=[0, \infty)$ endowed with the dislocated metric $\rho(\xi, \eta)=\max \{x, y\}$ for all $\xi, \eta \in[0, \infty)$. Define $T$ : $M \rightarrow M$ and $\alpha: M \times M \rightarrow \mathbb{R}_{0}^{+}$by

$$
\begin{gathered}
T \xi= \begin{cases}\frac{1}{2}\left(\xi-\frac{1}{2}\right), & \xi>1, \\
0, & 0 \leq \xi \leq 1,\end{cases} \\
\alpha(\xi, \eta)= \begin{cases}1, & \text { if } \xi, \eta \in[0,1], \\
0, & \text { otherwise. }\end{cases}
\end{gathered}
$$

Clearly, $T$ is not continuous at 1 which shows that Theorem 7 is not applicable in this case.

We will prove that a self-mapping $T$ is $(\alpha-\psi)$-MKC. Let $\varepsilon>0$ be given. Take $\delta>0$ and suppose that $\varepsilon \leq \psi(\rho(\xi, \eta))<$ $\varepsilon+\delta$; we want to show that

$$
\alpha(\xi, \eta) \rho(T \xi, T \eta)<\varepsilon
$$

Suppose that $\alpha(\xi, \eta)=1$; then $\xi, \eta \in[0,1]$ and so $T \xi=$ $0, T \eta=0$. Hence,

$$
\rho(T \xi, T \eta)=\rho(0,0)=\max \{0,0\}=0<\varepsilon .
$$

Also, $T$ is $\alpha$-admissible. To see this, let $\alpha(\xi, \eta) \geq 1$; then, both $\xi, \eta \in[0,1]$. Due to the definition of $T$, we have $T \xi=$ $0 \in[0,1]$ and $T \eta=0 \in[0,1]$. Thus, we get $\alpha(T \xi, T \eta) \geq 1$.

Moreover, there exists $\xi_{0} \in M$ such that $\alpha\left(\xi_{0}, T \xi_{0}\right) \geq 1$. Indeed, for $\xi_{0}=1 / 2$, we have

$$
\alpha\left(\frac{1}{2}, T \frac{1}{2}\right)=\alpha\left(\frac{1}{2}, 0\right)=1
$$

Finally, let $\left\{\xi_{n}\right\}$ be a sequence in $M$ such that $\alpha\left(\xi_{n}, \xi_{n+1}\right) \geq$ 1 for all $n$ and $\xi_{n} \rightarrow \xi \in M$ as $n \rightarrow \infty$. Since $\alpha\left(\xi_{n}, \xi_{n+1}\right) \geq 1$ for all $n$, by the definition of $\alpha$, we have $\xi_{n} \in[0,1]$ for all $n$ and $\xi \in[0,1]$; then, $\alpha\left(\xi_{n}, \xi\right)=1$.

So, we conclude that all hypotheses of Theorem 8 are fulfilled. Hence, we proved that $T$ has a fixed point.

\subsection{Generalized-( $\alpha, \psi)$-Meir-Keeler Contractive Mapping}

Definition 11. Suppose that $(M, \rho)$ is a DMS. A self-mapping $T: M \rightarrow M$ is said to be generalized- $(\alpha, \psi)$-Meir-Keeler contractive (in brief, generalized- $(\alpha, \psi)$-MKC) if there exist $\psi \in \Psi$ and $\alpha: M \times M \rightarrow \mathbb{R}_{0}^{+}$such that, for each $\varepsilon>0$, there exists $\delta>0$ such that

$$
\begin{aligned}
& \varepsilon \leq \psi\left(\max _{T}(\xi, \eta)\right)<\varepsilon+\delta \\
& \text { implies } \alpha(\xi, \eta) \rho(T \xi, T \eta)<\varepsilon,
\end{aligned}
$$

where

$$
\begin{aligned}
& \max _{T}(\xi, \eta)=\max \{\rho(\xi, \eta), \rho(\xi, T \xi), \rho(\eta, T \eta), \\
& \left.\quad \frac{1}{4}(\rho(\xi, T \eta)+\rho(\eta, T \xi))\right\} .
\end{aligned}
$$

If a self-mapping $T: M \rightarrow M$ is a generalized- $(\alpha, \psi)$ MKC, then we have

$$
\alpha(\xi, \eta) \rho(T \xi, T \eta) \leq \psi\left(\max _{T}(\xi, \eta)\right)
$$


Very recently, Popescu [7] improved the triangular $\alpha$ admissible notion as follows.

Definition 12 (see [7]). Suppose that $T: M \rightarrow M$ is a selfmapping and $\alpha: M \times M \rightarrow \mathbb{R}_{0}^{+}$is a function. A self-mapping $T$ is called $\alpha$-orbital admissible if

$$
\begin{gathered}
(T 3) \alpha(\xi, T \xi) \geq 1 \Longrightarrow \\
\alpha\left(T \xi, T^{2} \xi\right) \geq 1 .
\end{gathered}
$$

A self-mapping $T$ is called triangular $\alpha$-orbital admissible if $T$ is $\alpha$-orbital admissible and

$$
\begin{gathered}
(T 4) \alpha(\xi, \eta) \geq 1, \alpha(\eta, T \eta) \geq 1 \Longrightarrow \\
\alpha(\xi, T \eta) \geq 1 .
\end{gathered}
$$

As it was mentioned in [7], every (triangular) $\alpha$ admissible function is (triangular) $\alpha$-orbital admissible function. The converse is false; see, for example, [7, Example 7].

Lemma 13 (see [7]). Suppose that $T: M \rightarrow M$ is a triangular $\alpha$-orbital admissible mapping. Suppose also that there exists $\xi_{0} \in M$ such that $\alpha\left(\xi_{0}, T \xi_{0}\right) \geq 1$. Define an iterative sequence $\left\{\xi_{n}\right\}$ by $\xi_{n+1}=T \xi_{n}$ for each $n \in \mathbb{N}_{0}$. Then, one has $\alpha\left(\xi_{n}, \xi_{m}\right) \geq 1$ for all $m, n \in \mathbb{N}$ with $n<m$.

The following is the first main result of this section.

Theorem 14. Suppose that $(M, \rho)$ is a complete DMS, a selfmapping $T: M \rightarrow M$ is a generalized- $(\alpha, \psi)-M K C$, and the following conditions are fulfilled:

(i) $T$ is triangular $\alpha$-orbital admissible mapping.

(ii) There exists $\xi_{0} \in M$ such that $\alpha\left(\xi_{0}, T \xi_{0}\right) \geq 1$.

(iii) $T$ is continuous.

Then, $T$ has a fixed point; that is, there exists $\theta \in M$ such that $T \theta=\theta$.

Proof. Take $\xi_{0} \in M$ such that $\alpha\left(\xi_{0}, T \xi_{0}\right) \geq 1$. As in the proof of Theorem 7, we define an iterative sequence $\left\{\xi_{n}\right\}$ in $M$ as

$$
\xi_{n+1}=T \xi_{n}, \quad \text { for each } n \in \mathbb{N}_{0} .
$$

Without loss of generality, we assume that $\xi_{n} \neq \xi_{n+1}$, for all $n$; then,

$$
\rho\left(\xi_{n}, \xi_{n+1}\right)>0, \quad \forall n \in \mathbb{N}
$$

Indeed, if there exists some $n_{0} \in \mathbb{N}$ such that $\xi_{n_{0}}=\xi_{n_{0}+1}$, then the proof is completed since $u=\xi_{n_{0}}=\xi_{n_{0}+1}=T \xi_{n_{0}}=$ $T u$.

Since $T$ is triangular $\alpha$-orbital admissible mapping, by using Lemma 13, we derive that

$$
\alpha\left(\xi_{n}, \xi_{m}\right) \geq 1, \quad \forall m, n \in \mathbb{N} \text { with } n<m .
$$

Step 1. We will prove that

$$
\lim _{n \rightarrow \infty} \rho\left(\xi_{n}, \xi_{n+1}\right)=0 .
$$

Taking (47) and (48) into account together with the fact that $T$ is a generalized- $(\alpha, \psi)$-MKC mapping, for each $n \in \mathbb{N}$, we derive

$$
\begin{aligned}
\rho\left(\xi_{n}, \xi_{n+1}\right) & =\rho\left(T \xi_{n-1}, T \xi_{n}\right) \\
& \leq \alpha\left(\xi_{n-1}, \xi_{n}\right) \rho\left(T \xi_{n-1}, T \xi_{n}\right) \\
& \leq \psi\left(\max _{T}\left(\xi_{n-1}, \xi_{n}\right)\right)<\max _{T}\left(\xi_{n-1}, \xi_{n}\right),
\end{aligned}
$$

where

$$
\begin{aligned}
& \max _{T}\left(\xi_{n-1}, \xi_{n}\right)=\max \left\{\rho\left(\xi_{n-1}, \xi_{n}\right), \rho\left(\xi_{n-1}, T \xi_{n-1}\right),\right. \\
& \left.\rho\left(\xi_{n}, T \xi_{n}\right), \frac{1}{4}\left[\rho\left(\xi_{n-1}, T \xi_{n}\right)+\rho\left(\xi_{n}, T \xi_{n-1}\right)\right]\right\} \\
& \quad=\max \left\{\rho\left(\xi_{n-1}, \xi_{n}\right), \rho\left(\xi_{n}, \xi_{n+1}\right),\right. \\
& \left.\quad \frac{1}{4}\left[\rho\left(\xi_{n-1}, \xi_{n+1}\right)+\rho\left(\xi_{n}, \xi_{n}\right)\right]\right\} .
\end{aligned}
$$

Regarding $\left(\rho_{3}\right)$, we estimate the last term in the expression $\max _{T}\left(\xi_{n-1}, \xi_{n}\right)$ as follows:

$$
\begin{aligned}
& \frac{1}{4}\left[\rho\left(\xi_{n-1}, \xi_{n+1}\right)+\rho\left(\xi_{n}, \xi_{n}\right)\right] \\
& \quad \leq \max \left\{\rho\left(\xi_{n-1}, \xi_{n}\right), \rho\left(\xi_{n}, \xi_{n+1}\right)\right\} .
\end{aligned}
$$

Consequently, we get

$$
\max _{T}\left(\xi_{n-1}, \xi_{n}\right)=\max \left\{\rho\left(\xi_{n-1}, \xi_{n}\right), \rho\left(\xi_{n}, \xi_{n+1}\right)\right\} .
$$

Let us examine two cases. Let $\max \left\{\rho\left(\xi_{n-1}, \xi_{n}\right), \rho\left(\xi_{n}\right.\right.$, $\left.\left.\xi_{n+1}\right)\right\}=\rho\left(\xi_{n}, \xi_{n+1}\right)$. Since $\psi$ is nondecreasing, from (50), we get

$$
\rho\left(\xi_{n}, \xi_{n+1}\right) \leq \psi\left(\rho\left(\xi_{n}, \xi_{n+1}\right)\right)<\rho\left(\xi_{n}, \xi_{n+1}\right),
$$

which is a contradiction. Thus, $\max \left\{\rho\left(\xi_{n-1}, \xi_{n}\right), \rho\left(\xi_{n}, \xi_{n+1}\right)\right\}=$ $\rho\left(\xi_{n-1}, \xi_{n}\right)$ and again, by (50), we find

$$
\rho\left(\xi_{n}, \xi_{n+1}\right) \leq \psi\left(\rho\left(\xi_{n-1}, \xi_{n}\right)\right)<\rho\left(\xi_{n-1}, \xi_{n}\right),
$$

$\forall n \in \mathbb{N}$

So, we deduce that the sequence $\left\{\rho\left(\xi_{n}, \xi_{n+1}\right)\right\}$ is nonincreasing and bounded below by zero.

Hence, there exists $t \in[0, \infty)$ such that

$$
\lim _{n \rightarrow \infty} \rho\left(\xi_{n}, \xi_{n+1}\right)=t .
$$

Recursively, we derive from (55) that

$$
\rho\left(\xi_{n}, \xi_{n+1}\right) \leq \psi^{n}\left(\rho\left(\xi_{0}, \xi_{1}\right)\right), \quad \forall n,
$$

by keeping in mind that $\psi$ is nondecreasing.

On account of (57) and $\left(\Psi_{1}\right)$, we obtain

$$
\lim _{n \rightarrow \infty} \rho\left(\xi_{n}, \xi_{n+1}\right)=0 .
$$


It is obvious from the triangle inequality that

$$
\lim _{n \rightarrow \infty} \rho\left(\xi_{n}, \xi_{n}\right) \leq 2 \lim _{n \rightarrow \infty} \rho\left(\xi_{n}, \xi_{n+1}\right)=0 .
$$

Step 2. We will prove that $\left\{\xi_{n}\right\}$ is a Cauchy sequence.

Suppose, on the contrary, that there exist $\varepsilon>0$ and a subsequence $\left\{\xi_{n(i)}\right\}$ of $\left\{\xi_{n}\right\}$ such that

$$
\rho\left(\xi_{n(i)}, \xi_{n(i+1)}\right)>2 \varepsilon .
$$

First, we will show the existence of $k \in \mathbb{N}$ such that $n(i)<$ $k \leq n(i+1)$. Later, we will prove that, for given $\varepsilon>0$ above, there exists $\delta>0$ such that

$$
\begin{aligned}
& \varepsilon \leq \psi\left(\max _{T}\left(\xi_{n(i)}, \xi_{k}\right)\right)<\varepsilon+\delta \\
& \text { but } \alpha\left(\xi_{n(i)}, \xi_{k}\right) \rho\left(T \xi_{n(i)}, T \xi_{k}\right) \geq \varepsilon,
\end{aligned}
$$

which contradicts (60), where

$$
\begin{gathered}
\max _{T}\left(\xi_{n(i)}, \xi_{k}\right)=\max \left\{\rho\left(\xi_{n(i)}, \xi_{k}\right), \rho\left(\xi_{n(i)}, \xi_{n(i)+1}\right),\right. \\
\left.\rho\left(\xi_{k}, \xi_{k+1}\right), \frac{1}{4}\left[\rho\left(\xi_{n(i)}, \xi_{k+1}\right)+\rho\left(\xi_{k}, \xi_{n(i)+1}\right)\right]\right\} .
\end{gathered}
$$

Let $r=\min \{\varepsilon, \delta\}$. On account of Step 1, we will choose $n_{0} \in \mathbb{N}$ in a way that

$$
\begin{aligned}
d_{n} & =\rho\left(\xi_{n}, \xi_{n+1}\right)<\frac{r}{4}, \\
\rho\left(\xi_{n}, \xi_{n}\right) & <\frac{r}{2},
\end{aligned}
$$

for all $n \geq n_{0}$. Due to our construction, clearly, we have $n(i) \leq$ $n(i+1)-1$. If $\rho\left(\xi_{n(i)}, \xi_{n(i+1)-1}\right) \leq \varepsilon+r / 2$, then, by using $\left(\rho_{3}\right)$, we have

$$
\begin{aligned}
\rho\left(\xi_{n(i)}, \xi_{n(i+1)}\right) \leq & \rho\left(\xi_{n(i)}, \xi_{n(i+1)-1}\right) \\
& +\rho\left(\xi_{n(i+1)-1}, \xi_{n(i+1)}\right) \\
\leq & \varepsilon+\frac{r}{2}+d_{n(i+1)-1}<\varepsilon+\frac{r}{2}+\frac{r}{4} \\
= & \varepsilon+\frac{3 r}{4}<2 \varepsilon,
\end{aligned}
$$

which is a contradiction due to (60). Consequently, there are values of $k \in \mathbb{N}$ such that $n(i) \leq k \leq n(i+1)$ and $\rho\left(\xi_{n(i)}, \xi_{k}\right)>$ $\varepsilon+r / 2$. Indeed, if

$$
\rho\left(\xi_{n(i)}, \xi_{n(i)+1}\right) \geq \varepsilon+\frac{r}{2},
$$

then we obtain

$$
d_{n(i)}=\rho\left(\xi_{n(i)}, \xi_{n(i)+1}\right) \geq \varepsilon+\frac{r}{2} \geq r+\frac{r}{2}>\frac{r}{4}
$$

which contradicts (63).

Moreover, the case

$$
\rho\left(\xi_{n(i)}, \xi_{n(i)}\right) \geq \varepsilon+\frac{r}{2}
$$

is impossible due to (64).
Hence, we choose the smallest integer $k$ with $k>n(i)$ in a way that

$$
\rho\left(\xi_{n(i)}, \xi_{k}\right) \geq \varepsilon+\frac{r}{2}
$$

So, necessarily, we have $\rho\left(\xi_{n(i)}, \xi_{k-1}\right)<\varepsilon+r / 2$. Hence, we find that

$$
\begin{aligned}
\rho\left(\xi_{n(i)}, \xi_{k}\right) & \leq \rho\left(\xi_{n(i)}, \xi_{k-1}\right)+\rho\left(\xi_{k-1}, \xi_{k}\right)<\varepsilon+\frac{r}{2}+\frac{r}{4} \\
& =\varepsilon+\frac{3 r}{4},
\end{aligned}
$$

and, hence, we get the following approximation:

$$
\varepsilon+\frac{r}{2} \leq \rho\left(\xi_{n(i)}, \xi_{k}\right)<\varepsilon+\frac{3 r}{4},
$$

for a natural number $k$ satisfying $n(i)<k \leq n(i+1)$.

Thus, the first three terms of (62) are bounded above by $\varepsilon+r$; that is,

$$
\begin{gathered}
\rho\left(\xi_{n(i)}, \xi_{k}\right)<\varepsilon+\frac{3 r}{4}<\varepsilon+r, \\
\rho\left(\xi_{n(i)}, \xi_{n(i)+1}\right)=d_{n(i)}<\frac{r}{4}<\varepsilon+r, \\
\rho\left(\xi_{k}, \xi_{k+1}\right)=d_{k}<\frac{r}{4}<\varepsilon+r .
\end{gathered}
$$

Eventually, the last terms of (62) can be estimated from above as follows:

$$
\begin{aligned}
& \frac{1}{4}\left[\rho\left(\xi_{n(i)}, \xi_{k+1}\right)+\rho\left(\xi_{n(i)+1}, \xi_{k}\right)\right] \leq \frac{1}{4}\left[\rho\left(\xi_{n(i)}, \xi_{k}\right)\right. \\
& \left.+\rho\left(\xi_{k}, \xi_{k+1}\right)+\rho\left(\xi_{n(i)+1}, \xi_{n(i)}\right)+\rho\left(\xi_{n(i)}, \xi_{k}\right)\right] \leq \frac{1}{2} \\
& \quad \rho\left(\xi_{n(i)}, \xi_{k}\right)+\frac{1}{4}\left[d_{k}+d_{n(i)}\right] \leq \rho\left(\xi_{n(i)}, \xi_{k}\right)+\frac{1}{4}\left[d_{k}\right. \\
& \left.+d_{n(i)}\right]<\varepsilon+\frac{3 r}{4}+\frac{1}{4}\left[\frac{2 r}{4}\right]=\varepsilon+\frac{7 r}{8}<\varepsilon+r .
\end{aligned}
$$

Combining estimations (72), (73), (74), and (75), we conclude that

$$
\psi\left(\max _{T}\left(\xi_{n(i)}, \xi_{k}\right)\right) \leq \max _{T}\left(\xi_{n(i)}, \xi_{k}\right)<\varepsilon+r \leq \varepsilon+\delta .
$$

Since $T$ is generalized- $(\alpha-\psi)$-MKC mapping and since it is triangular $\alpha$-orbital admissible mapping, we have

$$
\rho\left(\xi_{n(i)+1}, \xi_{k+1}\right) \leq \alpha\left(\xi_{n(i)}, \xi_{k}\right) \rho\left(\xi_{n(i)+1}, \xi_{k+1}\right)<\varepsilon .
$$

On the other hand, by $\left(\rho_{3}\right)$, we have

$$
\begin{aligned}
\rho\left(\xi_{n(i)}, \xi_{k}\right) \leq & \rho\left(\xi_{n(i)}, \xi_{n(i)+1}\right)+\rho\left(\xi_{n(i)+1}, \xi_{k}\right) \\
\leq & \rho\left(\xi_{n(i)}, \xi_{n(i)+1}\right)+\rho\left(\xi_{n(i)+1}, \xi_{k+1}\right) \\
& +\rho\left(\xi_{k+1}, \xi_{k}\right)<\frac{r}{4}+\varepsilon+\frac{r}{4}=\varepsilon+\frac{r}{2},
\end{aligned}
$$

which is a contradiction with (71). 
Thus, claim (60) is false and the constructive sequence $\left\{\xi_{n}\right\}$ is Cauchy; that is,

$$
\lim _{n, m \rightarrow \infty} \rho\left(\xi_{n}, \xi_{m}\right)=0 .
$$

Since $(M, \rho)$ is a complete DMS, then there exists $\theta \in M$ such that

$$
\lim _{n \rightarrow \infty} \rho\left(\xi_{n}, \theta\right)=\rho(\theta, \theta)=\lim _{n, m \rightarrow \infty} \rho\left(\xi_{n}, \xi_{m}\right)=0 .
$$

On account of the continuity of the self-mapping $T$, we deduce that

$$
\begin{aligned}
\lim _{n \rightarrow \infty} \rho\left(\xi_{n+1}, T \theta\right) & =\lim _{n \rightarrow \infty} \rho\left(T \xi_{n}, T \theta\right)=\rho(T \theta, T \theta) \\
& =\lim _{n, m \rightarrow \infty} \rho\left(T \xi_{n}, T \xi_{m}\right)=0 .
\end{aligned}
$$

Since $\xi_{n+1} \rightarrow \theta$ as $n \rightarrow \infty$ and $\rho(\theta, \theta)=0$, then, by using Lemma 4 , we get

$$
\lim _{n \rightarrow \infty} \rho\left(\xi_{n+1}, T \theta\right)=\rho(\theta, T \theta) .
$$

Regarding (81) and (82), we get $\rho(\theta, T \theta)=0$. Thus, by $\left(\rho_{1}\right)$, we conclude that $T \theta=\theta$.

Theorem 15. Suppose that $(M, \rho)$ is a complete DMS, a selfmapping $T: M \rightarrow M$ is a generalized- $(\alpha, \psi)-M K C$, and the following conditions are fulfilled:

(i) $T$ is triangular $\alpha$-orbital admissible mapping.

(ii) There exists $\xi_{0} \in M$ such that $\alpha\left(\xi_{0}, T \xi_{0}\right) \geq 1$.

(iii) If $\left\{\xi_{n}\right\}$ is a sequence in $M$ such that $\alpha\left(\xi_{n}, \xi_{n+1}\right) \geq 1$ for all $n$ and $\xi_{n} \rightarrow \xi \in M$ as $n \rightarrow \infty$, then $\alpha\left(\xi_{n}, \xi\right) \geq 1$ for all $n$.

Then, $T$ has a fixed point; that is, there exists $\theta \in M$ such that $T \theta=\theta$.

Proof. Define an iterative sequence $\left\{\xi_{n}\right\}$ in $M$ as in Theorem 14. Suppose that there is $k_{0} \in \mathbb{N}$ such that $\xi_{k_{0}}=\xi_{k_{0}+1}$; then, the proof is completed since $u=\xi_{k_{0}}=\xi_{k_{0}+1}=T \xi_{k_{0}}=$ $T u$. So, it is interesting to assume that $\xi_{n} \neq \xi_{n+1}$, for all $n \in \mathbb{N}_{0}$. Consequently, we have

$$
\rho\left(\xi_{n}, \xi_{n+1}\right)>0, \quad \forall n \in \mathbb{N}_{0} .
$$

From (48), we find that

$$
\alpha\left(\xi_{n}, \xi_{n+1}\right) \geq 1, \quad \forall n \in \mathbb{N}_{0} .
$$

Following the lines for the proofs of Steps 1 and 2 in Theorem 14, we derive that $\left\{\xi_{n}\right\}$ is a Cauchy sequence and

$$
\lim _{n, m \rightarrow \infty} \rho\left(\xi_{n}, \xi_{m}\right)=0 \text {. }
$$

Since $(M, \rho)$ is a complete DMS, then there exists $\theta \in M$ such that

$$
\lim _{n \rightarrow \infty} \rho\left(\xi_{n}, \theta\right)=\rho(\theta, \theta)=\lim _{n, m \rightarrow \infty} \rho\left(\xi_{n}, \xi_{m}\right)=0 .
$$

We will prove that $\theta=T \theta$. Suppose, on the contrary, that $\rho(\theta, T \theta)>0$.

Notice from (84), (86), and (iii) that

$$
\alpha\left(\xi_{n}, \theta\right) \geq 1, \quad \forall n
$$

By using $\left(\rho_{3}\right)$ and (87) together with the assumption of the theorem that $T$ is a generalized- $(\alpha-\psi)$-MKC mapping, we obtain

$$
\begin{aligned}
\rho(T \theta, \theta) & \leq \rho\left(T \theta, T \xi_{n}\right)+\rho\left(\xi_{n+1}, \theta\right) \\
& \leq \alpha\left(\xi_{n}, \theta\right) \rho\left(T \xi_{n}, T \theta\right)+\rho\left(\xi_{n+1}, \theta\right) \\
& \leq \psi\left(\max _{T}\left(\xi_{n}, \theta\right)\right)+\rho\left(\xi_{n+1}, \theta\right),
\end{aligned}
$$

where

$$
\begin{aligned}
& \max _{T}\left(\xi_{n}, \theta\right)=\max \left\{\rho\left(\xi_{n}, \theta\right), \rho\left(\xi_{n}, \xi_{n+1}\right), \rho(\theta, T \theta),\right. \\
& \left.\quad \frac{1}{4}\left[\rho\left(\xi_{n}, T \theta\right)+\rho\left(\xi_{n+1}, \theta\right)\right]\right\} .
\end{aligned}
$$

Notice that as $\rho(\theta, T \theta)>0$, then we must have $\max _{T}\left(\xi_{n}\right.$, $\theta)>0$.

Regarding $\left(\rho_{3}\right)$, we have

$$
\begin{aligned}
& \frac{1}{4}\left[\rho\left(\xi_{n}, T \theta\right)+\rho\left(\xi_{n+1}, \theta\right)\right] \\
& \quad \leq \max \left\{\rho\left(\xi_{n}, \theta\right), \rho\left(\xi_{n}, \xi_{n+1}\right), \rho(\theta, T \theta)\right\} .
\end{aligned}
$$

By the above inequality, we have

$$
\max _{T}\left(\xi_{n}, \theta\right)=\max \left\{\rho\left(\xi_{n}, \theta\right), \rho\left(\xi_{n}, \xi_{n+1}\right), \rho(\theta, T \theta)\right\} .
$$

Suppose that $\max _{T}\left(\xi_{n}, \theta\right)=\rho\left(\xi_{n}, \theta\right)$; then, from (88), we get

$$
\begin{aligned}
\rho(T \theta, \theta) & \leq \psi\left(\rho\left(\xi_{n}, \theta\right)\right)+\rho\left(\xi_{n+1}, \theta\right) \\
& <\rho\left(\xi_{n}, \theta\right)+\rho\left(\xi_{n+1}, \theta\right) .
\end{aligned}
$$

Taking $n \rightarrow \infty$ in (92), we have

$$
\rho(T \theta, \theta)<\rho(\theta, \theta)+\rho(\theta, \theta)=0,
$$

which is a contradiction.

Now, we suppose that $\max _{T}\left(\xi_{n}, \theta\right)=\rho\left(\xi_{n}, \xi_{n+1}\right)$; then, by (88), we find that

$$
\begin{aligned}
\rho(T \theta, \theta) & \leq \psi\left(\rho\left(\xi_{n}, \xi_{n+1}\right)\right)+\rho\left(\xi_{n+1}, \theta\right) \\
& <\rho\left(\xi_{n}, \xi_{n+1}\right)+\rho\left(\xi_{n+1}, \theta\right) .
\end{aligned}
$$

Letting $n \rightarrow \infty$, this implies that

$$
\rho(T \theta, \theta)<\lim _{n \rightarrow \infty}\left[\rho\left(\xi_{n}, \xi_{n+1}\right)+\rho\left(\xi_{n+1}, \theta\right)\right]=0,
$$

which is again a contradiction. 
Finally, we suppose that $\max _{T}\left(\xi_{n}, \theta\right)=\rho(\theta, T \theta)$; then, again from (88), we have

$$
\rho(T \theta, \theta) \leq \psi(\rho(\theta, T \theta))+\rho\left(\xi_{n+1}, \theta\right) .
$$

Letting $n \rightarrow \infty$, in the above inequality, we get

$$
\begin{aligned}
\rho(T \theta, \theta) & \leq \psi(\rho(\theta, T \theta))+\rho(\theta, \theta) \\
& <\rho(\theta, T \theta)+\rho(\theta, \theta)=\rho(T \theta, \theta),
\end{aligned}
$$

so we also have a contradiction. Thus, we have $\rho(T \theta, \theta)=0$ and, by $\left(\rho_{1}\right)$, we have $T \theta=\theta$.

2.3. The Uniqueness of the Fixed Point. We propose the following conditions for the uniqueness of the fixed points of the mappings discussed in Sections 2.1 and 2.2. Let Fix $(T)$ denote the set of fixed points of the mapping $T$.

We, first, recollect the following interesting condition for uniqueness of a fixed point of an $(\alpha-\psi)$-MKC mapping.

(H) For all $\xi, \eta \in \operatorname{Fix}(T)$, there exists $\zeta \in M$ such that $\alpha(\xi, \zeta) \geq 1$ and $\alpha(\eta, \zeta) \geq 1$, where we have the following.

Theorem 16. Putting condition $(H)$ to the statements of Theorem 7 (resp., Theorem 8), one obtains that $\theta$ is the unique fixed point of $T$.

Proof. Let $\theta$ and $\nu$ be two distinct fixed points of $T$. From $(\mathrm{H})$, there exists $\zeta \in M$ such that

$$
\begin{aligned}
& \alpha(\theta, \zeta) \geq 1 \\
& \alpha(\nu, \zeta) \geq 1 .
\end{aligned}
$$

Due to the fact that $T$ is $\alpha$-admissible, we have

$$
\begin{aligned}
& \alpha(\theta, T \zeta) \geq 1 \\
& \alpha(\nu, T \zeta) \geq 1
\end{aligned}
$$

Inductively, we obtain

$$
\begin{aligned}
& \alpha\left(\theta, T^{n} \zeta\right) \geq 1, \\
& \alpha\left(\nu, T^{n} \zeta\right) \geq 1,
\end{aligned}
$$

$\forall n \in \mathbb{N}$.

From the above relation and since $T$ is an $(\alpha, \psi)$-MKC mapping, we have

$$
\begin{aligned}
\rho\left(\theta, T^{n} \zeta\right) & \leq \alpha\left(\theta, T^{n-1} \zeta\right) \rho\left(T \theta, T^{n} \zeta\right) \\
& \leq \psi\left(\rho\left(\theta, T^{n-1} \zeta\right)\right)
\end{aligned}
$$

Iteratively, we get

$$
\rho\left(\theta, T^{n} \zeta\right) \leq \psi^{n}(\rho(\theta, \zeta)) .
$$

Letting $n \rightarrow \infty$, we obtain

$$
\lim _{n \rightarrow \infty} \rho\left(\theta, T^{n} \zeta\right)=0 .
$$

Similarly, we can prove that

$$
\lim _{n \rightarrow \infty} \rho\left(\nu, T^{n} \zeta\right)=0
$$

Using $\left(\rho_{3}\right)$, we have

$$
\rho(\theta, \nu) \leq \rho\left(\theta, T^{n} \zeta\right)+\rho\left(T^{n} \zeta, \nu\right) .
$$

Taking $n \rightarrow \infty$, we find that

$$
\rho(\theta, v)=0,
$$

and so, by $\left(\rho_{1}\right), \theta=\nu$.

As an alternative uniqueness condition for fixed points of $(\alpha-\psi)$-MKC mapping, we suggest the following hypothesis:

(U) For all $\xi, \eta \in \operatorname{Fix}(T)$, then $\alpha(\xi, \eta) \geq 1$.

Theorem 17. Putting condition (U) to the statements of Theorem 7 (resp., Theorem 8), one finds that $\theta$ is the unique fixed point of $T$.

Proof. Let $\theta, v$ be two distinct fixed points of $T$. Then, by Lemma 4 part (C), we have

$$
\rho(\theta, v)>0 \text {. }
$$

Due to the property of $\psi\left(\Psi_{2}\right)$, we get

$$
\psi(\rho(\theta, \nu))>0
$$

Let $\varepsilon=\psi(\rho(\theta, \nu))$; then, for any $\delta>0$, we find that

$$
\varepsilon=\psi(\rho(\theta, \nu))<\varepsilon+\delta .
$$

Regarding (U) and the assumption of the theorem that $T$ is an $(\alpha, \psi)$-MKC mapping, we obtain

$$
\rho(\theta, \nu) \leq \alpha(\theta, \nu) \rho(T \theta, T \nu)<\psi(\rho(\theta, \nu))<\rho(\theta, \nu),
$$

which is a contradiction. Thus, $\theta=v$.

In what follows, we propose the conditions for the uniqueness of a fixed point of a generalized- $(\alpha-\psi)-\mathrm{MKC}$ mapping:

(H1) For all $\xi, \eta \in \operatorname{Fix}(T)$, there exists $\zeta \in M$ such that $\alpha(\xi, \zeta) \geq 1, \alpha(\eta, \zeta) \geq 1$, and $\alpha(\zeta, T \zeta) \geq 1$.

(H2) Let $\xi, \eta \in \operatorname{Fix}(T)$. If there exists a sequence $\left\{\zeta_{n}\right\}$ in $M$ such that $\alpha\left(\xi, \zeta_{n}\right) \geq 1, \alpha\left(\eta, \zeta_{n}\right) \geq 1$, and $\alpha\left(\zeta_{n}, \zeta_{n+1}\right) \geq$ 1 , then

$$
\rho\left(\zeta_{n}, \zeta_{n+1}\right) \leq \inf \left\{\rho\left(\xi, \zeta_{n}\right), \rho\left(\eta, \zeta_{n}\right)\right\}
$$

(H3) For any $\xi \in \operatorname{Fix}(T)$, then $\alpha(\xi, \xi) \geq 1$.

Theorem 18. Putting conditions (H1), (H2), and (H3) to the statements of Theorem 14 (resp., Theorem 15), one has that $\theta$ is the unique fixed point of $T$. 
Proof. Let $\theta, v$ be two distant fixed points of $T$. From (H1), there exists $\zeta \in M$ such that

$$
\begin{aligned}
\alpha(\theta, \zeta) & \geq 1, \\
\alpha(\nu, \zeta) & \geq 1, \\
\alpha(\zeta, T \zeta) & \geq 1 .
\end{aligned}
$$

Owing to the fact that $T$ is triangular $\alpha$-orbital admissible and $\alpha(\zeta, T \zeta) \geq 1$, by (T3), we have

$$
\alpha\left(T \zeta, T^{2} \zeta\right) \geq 1
$$

Inductively, we find

$$
\alpha\left(T^{n-1} \zeta, T^{n} \zeta\right) \geq 1, \quad \forall n \in \mathbb{N} .
$$

Now, since $\alpha(\theta, \zeta) \geq 1$ and $\alpha(\zeta, T \zeta) \geq 1$, then, by (T4), we deduce

$$
\alpha(\theta, T z) \geq 1
$$

Again, by (T4), since $\alpha(\theta, T \zeta) \geq 1$ and $\alpha\left(T \zeta, T^{2} \zeta\right) \geq 1$, we derive

$$
\alpha\left(\theta, T^{2} \zeta\right) \geq 1
$$

Inductively, we get

$$
\alpha\left(\theta, T^{n} \zeta\right) \geq 1, \quad \forall n \in \mathbb{N} .
$$

In an analogous way, we will prove that

$$
\alpha\left(\nu, T^{n} \zeta\right) \geq 1, \quad \forall n \in \mathbb{N} .
$$

Define an iterative sequence $\left\{\zeta_{n}\right\}$ by $\zeta_{n+1}=T \zeta_{n}$, for all $n \geq 0$ and $\zeta_{0}=\zeta$.

Step 1 . We will prove that

$$
\lim _{n \rightarrow \infty} \rho\left(\theta, \zeta_{n}\right)=0 .
$$

By (117) and the statement of the theorem that $T$ is generalized- $(\alpha, \psi)$-MKC mapping, we have

$$
\begin{aligned}
\rho\left(\theta, \zeta_{n+1}\right) & \leq \alpha\left(\theta, \zeta_{n}\right) \rho\left(T \theta, T \zeta_{n}\right) \\
& \leq \psi\left(\max _{T}\left(\theta, \zeta_{n}\right)\right) .
\end{aligned}
$$

If $\psi\left(\max _{T}\left(\theta, \zeta_{n}\right)\right)=0$, then

$$
\lim _{n \rightarrow \infty} \rho\left(\theta, \zeta_{n+1}\right)=0 .
$$

Now, suppose that $\psi\left(\max _{T}\left(\theta, \zeta_{n}\right)\right)>0$; then, $\max _{T}\left(\theta, \zeta_{n}\right)>0$. As $T$ is a generalized- $(\alpha, \psi)$-MKC mapping, we get

$$
\begin{aligned}
\rho\left(\theta, \zeta_{n+1}\right) & \leq \alpha\left(\theta, \zeta_{n}\right) \rho\left(T \theta, T \zeta_{n}\right) \leq \psi\left(\max _{T}\left(\theta, \zeta_{n}\right)\right) \\
& <\max _{T}\left(\theta, \zeta_{n}\right),
\end{aligned}
$$

where

$$
\begin{aligned}
& \max _{T}\left(\zeta_{n}, \theta\right)=\max \left\{\rho\left(\zeta_{n}, \theta\right), \rho\left(\zeta_{n}, \zeta_{n+1}\right), \rho(\theta, T \theta),\right. \\
& \left.\frac{1}{4}\left[\rho\left(\zeta_{n}, T \theta\right)+\rho\left(\zeta_{n+1}, \theta\right)\right]\right\} .
\end{aligned}
$$

Regarding (H2) and $\left(\rho_{3}\right)$, we have

$$
\max _{T}\left(\zeta_{n}, \theta\right)=\rho\left(\zeta_{n}, \theta\right) .
$$

Thus, we have

$$
\rho\left(\theta, \zeta_{n+1}\right)<\rho\left(\theta, \zeta_{n}\right) .
$$

Letting $n \rightarrow \infty$ in the inequality above, we obtain

$$
\lim _{n \rightarrow \infty} \rho\left(\theta, \zeta_{n+1}\right)<\lim _{n \rightarrow \infty} \rho\left(\theta, \zeta_{n}\right),
$$

which is a contradiction. Then,

$$
\max _{T}\left(\zeta_{n}, \theta\right)=\rho\left(\zeta_{n}, \theta\right)=0 .
$$

Hence, we get

$$
\lim _{n \rightarrow \infty} \rho\left(\theta, \zeta_{n}\right)=0 .
$$

Step 2. We will prove that

$$
\lim _{n \rightarrow \infty} \rho\left(\nu, \zeta_{n}\right)=0 .
$$

By (H3) and the assumption of the theorem that $T$ is a generalized- $(\alpha, \psi)$-MKC mapping, we find that

$$
\begin{aligned}
\rho(\nu, \nu) & \leq \alpha(\nu, \nu) \rho(T \nu, T \nu) \leq \psi\left(\max _{T}(\nu, \nu)\right) \\
& =\psi(\rho(\nu, \nu)) .
\end{aligned}
$$

Suppose, on the contrary, that $\rho(\nu, v)>0$. Then, from the above inequality, we obtain

$$
\rho(\nu, \nu) \leq \psi(\rho(\nu, \nu))<\rho(\nu, \nu),
$$

which is a contradiction. Thus, $\rho(\nu, \nu)=\rho(\nu, T \nu)=0$. of

In an analogous way of Step 1, we can complete the proof

$$
\lim _{n \rightarrow \infty} \rho\left(\nu, \zeta_{n}\right)=0 .
$$

By $\left(\rho_{3}\right)$, we have

$$
\begin{aligned}
\rho(\theta, \nu) & \leq \rho\left(\theta, T^{n-1} \zeta\right)+\rho\left(T^{n-1} \zeta, \nu\right) \\
& =\rho\left(\theta, \zeta_{n}\right)+\rho\left(\zeta_{n}, \nu\right) .
\end{aligned}
$$

Letting $n \rightarrow \infty$ in the above inequality, we find that

$$
\rho(\theta, \nu)=0 ;
$$

by $\left(\rho_{1}\right)$, we have $\theta=\nu$. 


\section{Ulam-Hyers Stability}

Definition 19 (see [8]). Let $(M, \rho)$ be a complete DMS and let $T: M \rightarrow M$ be a self-mapping. The fixed point inclusion

$$
\xi=T(\xi), \quad \xi \in M
$$

is called generalized Ulam-Hyers stable if and only if $\varsigma$ : $[0, \infty) \rightarrow[0, \infty)$ is increasing and continuous in 0 and $\varsigma(0)=$ 0 , such that, for each $\varepsilon>0$ and for each solution $\eta^{*} \in M$ of the equation

$$
\rho(y, T \eta) \leq \varepsilon
$$

there exists a solution $\xi^{*}$ of the fixed point inclusion (135) such that

$$
\rho\left(\eta^{*}, \xi^{*}\right) \leq \varsigma(\varepsilon)
$$

If there exists $c>0$ such that $\varsigma(t):=c \cdot t$, for each $t \in$ $[0, \infty)$, then the fixed point (135) is said to be Ulam-Hyers stable.

Theorem 20. Let $(M, \rho)$ be a complete DMS and let $T$ : $M \rightarrow M$ be a self-mapping. Suppose that all the hypotheses of Theorem 18 hold. Additionally, one supposes that

(i) $\psi(t)<t$ and the function $\beta: \mathbb{R}_{0}^{+} \rightarrow \mathbb{R}_{0}^{+}, \beta(r):=$ $r-\psi(r)$ is strictly increasing and onto;

(ii) for any solution $\eta^{*} \in M$ of (136) one has $\alpha\left(\xi^{*}, \eta^{*}\right) \geq 1$, where $\xi^{*} \in \operatorname{Fix}(T)$.

In these conditions, the fixed point inclusion (135) is generalized Ulam-Hyers stable.

Proof. We are in the conditions of Theorem 18; hence, there exists $\xi^{*} \in \operatorname{Fix}(T)$ such that $\rho\left(\xi^{*}, \xi^{*}\right)=0$. Let $\varepsilon>0$ and $\eta^{*}$ be a solution of (136).

From (ii), we have that $\alpha\left(\xi^{*}, \eta^{*}\right) \geq 1$, and because $T$ is $\alpha$ admissible we will obtain that $\alpha\left(T \xi^{*}, T \eta^{*}\right)=\alpha\left(\xi^{*}, T \eta^{*}\right) \geq 1$.

We have

$$
\begin{aligned}
\rho\left(\xi^{*}, \eta^{*}\right) & =\rho\left(T \xi^{*}, \eta^{*}\right) \\
\leq & \rho\left(T \xi^{*}, T \eta^{*}\right)+\rho\left(\eta^{*}, T \eta^{*}\right) \\
\leq & \rho\left(T \xi^{*}, T \eta^{*}\right)+\rho\left(\eta^{*}, T \eta^{*}\right) \\
\leq & \alpha\left(T \xi^{*}, T \eta^{*}\right) \rho\left(T \xi^{*}, T \eta^{*}\right) \\
& +\rho\left(\eta^{*}, T \eta^{*}\right) \leq \psi\left(\max _{T}\left(\xi^{*}, \eta^{*}\right)\right)+\varepsilon,
\end{aligned}
$$

where

$$
\begin{gathered}
\max _{T}\left(\xi^{*}, \eta^{*}\right)=\max \left\{\rho\left(\xi^{*}, \eta^{*}\right), \rho\left(\xi^{*}, T \xi^{*}\right),\right. \\
\left.\rho\left(\eta^{*}, T \eta^{*}\right), \frac{\rho\left(\xi^{*}, T \eta^{*}\right)+\rho\left(\eta^{*}, T \xi^{*}\right)}{4}\right\} .
\end{gathered}
$$

We have

$$
\begin{aligned}
& \frac{\rho\left(\xi^{*}, T \eta^{*}\right)+\rho\left(\eta^{*}, T \xi^{*}\right)}{4} \\
& \quad \leq \frac{1}{4}\left[\rho\left(\xi^{*}, \eta^{*}\right)+\rho\left(\eta^{*}, T \eta^{*}\right)+\rho\left(\eta^{*}, \xi^{*}\right)\right] \\
& \quad=\frac{1}{2} \rho\left(\xi^{*}, \eta^{*}\right)+\frac{1}{4} \rho\left(\eta^{*}, T \eta^{*}\right) \\
& \quad \leq \frac{1}{2} \rho\left(\xi^{*}, \eta^{*}\right)+\frac{1}{4} \varepsilon \leq \max \left\{\rho\left(\xi^{*}, \eta^{*}\right), \varepsilon\right\} .
\end{aligned}
$$

From here, we have

$$
\max _{T}\left(\xi^{*}, \eta^{*}\right) \leq \max \left\{\rho\left(\xi^{*}, \eta^{*}\right), \varepsilon\right\} .
$$

It is clear that if $\rho\left(\xi^{*}, \eta^{*}\right) \leq \varepsilon$ the proof is completed.

Suppose that $\max \left\{\rho\left(\xi^{*}, \eta^{*}\right), \varepsilon\right\}=\rho\left(\xi^{*}, \eta^{*}\right)$. Consequently, we have $\max _{T}\left(\xi^{*}, \eta^{*}\right) \leq \rho\left(\xi^{*}, \eta^{*}\right)$. On account of (138), we derive that

$$
\begin{aligned}
\rho\left(\xi^{*}, \eta^{*}\right) & \leq \psi\left(\rho\left(\xi^{*}, y^{*}\right)\right)+\varepsilon, \\
\rho\left(\xi^{*}, \eta^{*}\right)-\psi\left(\rho\left(\xi^{*}, \eta^{*}\right)\right) & \leq \varepsilon .
\end{aligned}
$$

From condition (i), we get that

$$
\beta\left(\rho\left(\xi^{*}, \eta^{*}\right)\right) \leq \varepsilon,
$$

and hence

$$
\rho\left(\xi^{*}, \eta^{*}\right) \leq \beta^{-1}(\varepsilon)
$$

Hence, (135) is generalized Ulam-Hyers stable.

Corollary 21. Suppose that $(M, \rho)$ is a complete DMS, $T$ : $M \rightarrow M$ is a self-mapping, and all the hypotheses of Theorem 16 hold. One supposes also that

(i) $\psi(t)<t$ and the function $\beta: \mathbb{R}_{0}^{+} \rightarrow \mathbb{R}_{0}^{+}, \beta(r):=r-$ $\psi(r)$ is strictly increasing and onto;

(ii) for any solution $\eta^{*} \in M$ of (136) one has $\alpha\left(\xi^{*}, \eta^{*}\right) \geq 1$, where $\xi^{*} \in \operatorname{Fix}(T)$.

In these conditions, the fixed point inclusion (135) is generalized Ulam-Hyers stable.

The proof is an analog of the proof of Theorem 20 .

\section{Conclusion}

It is possible to list several existing results as a consequence of our main results by choosing both auxiliary functions $\alpha$ and $\psi$ in a proper way like in the literature $[5,6,9-11]$ and so on.

\section{Competing Interests}

The authors declare that they have no competing interests. 


\section{Authors' Contributions}

All authors contributed equally and significantly to the writing of this paper. All authors read and approved the final paper.

\section{References}

[1] M. M. Fréchet, "Sur quelques points du calcul fonctionnel," Rendiconti del Circolo Matematico di Palermo, vol. 22, no. 1, pp. 1-72, 1906.

[2] P. Hitzler, Generalized metrics and topology in logic programming semantics [Ph.D. thesis], School of Mathematics, Applied Mathematics and Statistics, National University Ireland, University College Cork, Cork, Ireland, 2001.

[3] A. Amini-Harandi, "Metric-like spaces, partial metric spaces and fixed points," Fixed Point Theory and Applications, vol. 2012, article 204, 2012.

[4] E. Karapınar and P. Salimi, "Dislocated metric space to metric spaces with some fixed point theorems," Fixed Point Theory and Applications, vol. 2013, article 222, 2013.

[5] B. Samet, C. Vetro, and P. Vetro, "Fixed point theorems for $\alpha-\psi$ contractive type mappings," Nonlinear Analysis: Theory, Methods \& Applications, vol. 75, no. 4, pp. 2154-2165, 2012.

[6] H. Aydi, E. Karapınar, and B. Samet, "Fixed points for generalized $\alpha-\psi$-contractions on generalized metric spaces," Journal of Inequalities and Applications, vol. 2014, article 229, 2014.

[7] O. Popescu, "Some new fixed point theorems for $\alpha$-Geraghtycontraction type maps in metric spaces," Fixed Point Theory and Applications, vol. 2014, article 190, 2014.

[8] I. A. Rus, "Remarks on Ulam stability of the operatorial equations," Fixed Point Theory, vol. 10, no. 2, pp. 305-320, 2009.

[9] N. Bilgili and E. Karapinar, "A note on common fixed points for $(\psi, \alpha, \beta)$-weakly contractive mappings in generalized metric spaces," Fixed Point Theory and Applications, vol. 2013, article 287, 2013.

[10] A. Latif, M. E. Gordji, E. Karapinar, and W. Sintunavarat, "Fixed point results for generalized $(\alpha, \psi)$-Meir-Keeler contractive mappings and applications," Journal of Inequalities and Applications, vol. 2014, article 68, 2014.

[11] E. Karapınar and B. Samet, "Generalized $\alpha-\psi$ contractive type mappings and related fixed point theorems with applications," Abstract and Applied Analysis, vol. 2012, Article ID 793486, 17 pages, 2012. 


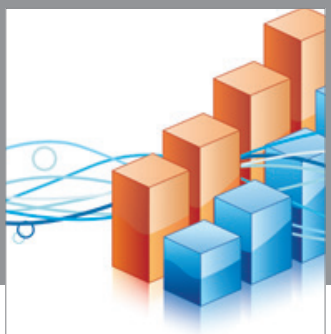

Advances in

Operations Research

vatem alat4

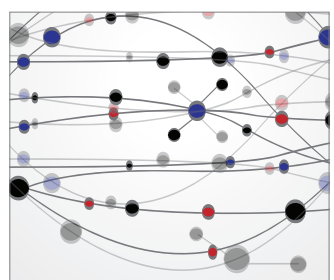

\section{The Scientific} World Journal
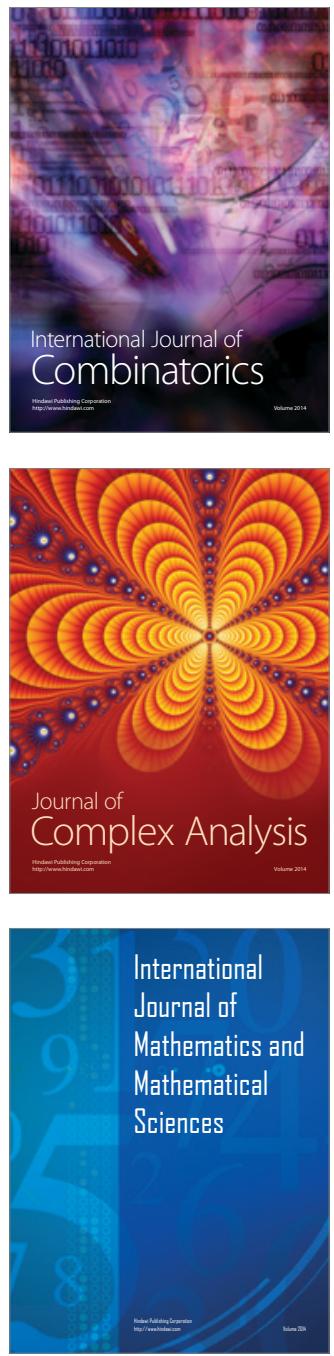
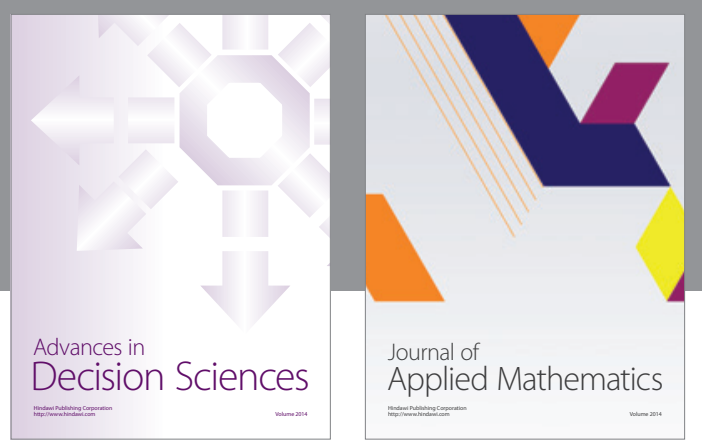

Algebra

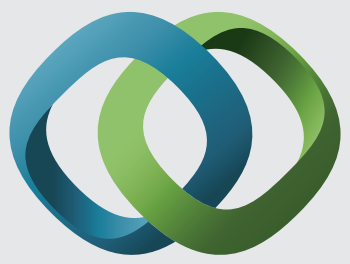

\section{Hindawi}

Submit your manuscripts at

http://www.hindawi.com
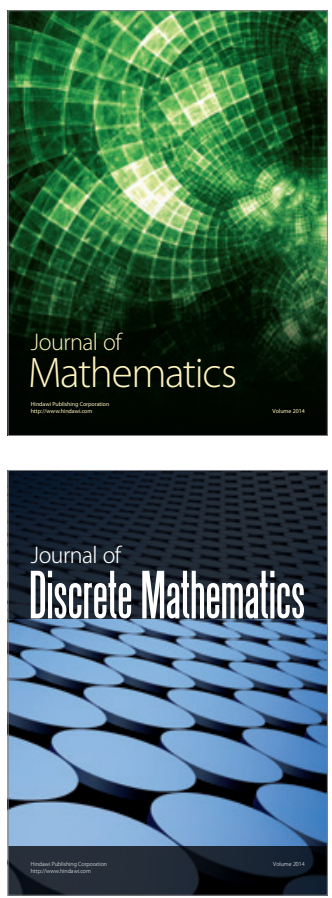

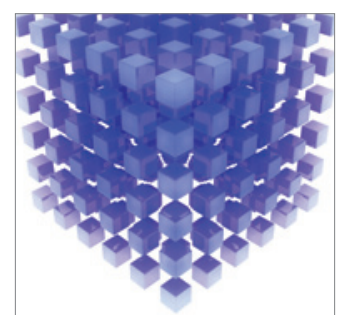

Mathematical Problems in Engineering
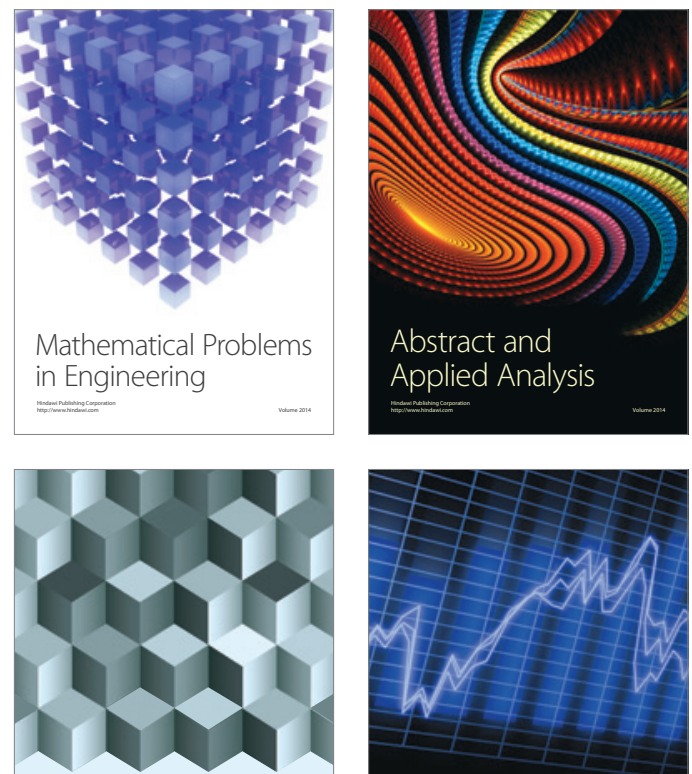

Journal of

Function Spaces

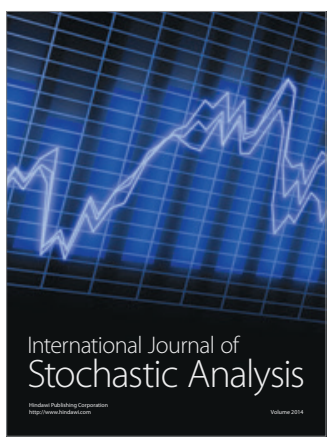

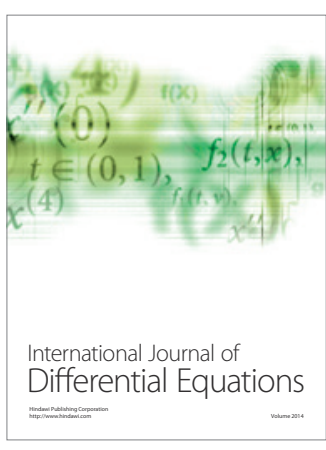
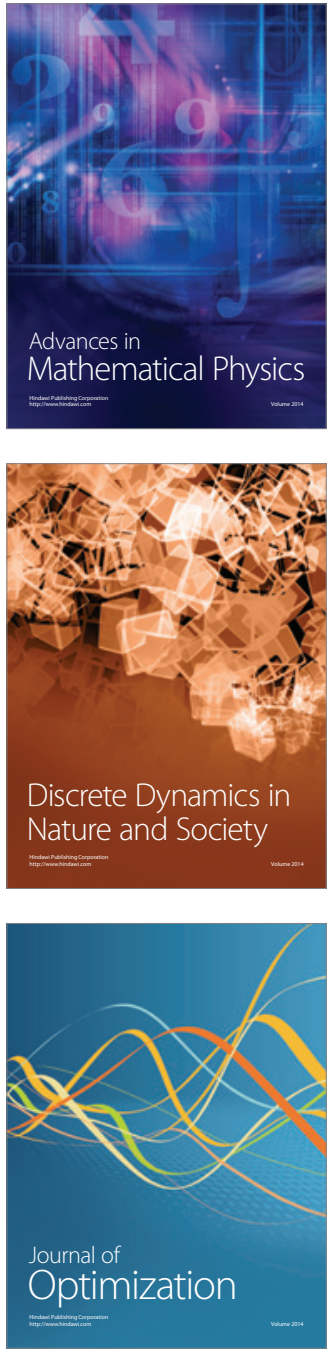